Cita bibliográfica: González Damiá, A. y Macías Ramírez, A. R. (2019). Influencia del capital social en la calidad de vida de los habitantes de la isla turística Cozumel, México Investigaciones Turísticas (18), pp. 138-168. http:// dx.doi.org/10.14198/INTURI2019.18.07

\title{
Influencia del capital social en la calidad de vida de los habitantes de la isla turística Cozumel, México
}

\section{Influence of social capital on the quality of life of the inhabitants of the tourist island Cozumel, Mexico}

\begin{abstract}
Alfonso González Damián (iD, Universidad de Quintana Roo, México alfonso.gonzalezdamian@gmail.com
\end{abstract}

Alma Rosa Macías Ramírez, Universidad de Quintana Roo, México macciasalma@hotmail.com

\section{RESUMEN}

Vivir en una isla de destino turístico, con oferta masificada de sol y playa, como es el caso de Cozumel, México, no es equivalente a vivir en el paraíso tal como lo ha señalado la literatura académica desde que se ha ocupado por analizar los impactos sociales, económicos, culturales y ambientales de la actividad. Existe evidencia empírica de que la percepción de beneficios económicos derivados del turismo impacta de manera positiva en la formación de capital social en la comunidad y también en la percepción de mejoría en la calidad de vida, sin embargo no abundan los estudios que profundicen en las relaciones entre el capital social y la calidad de vida, lo que se toma como objeto central del presente trabajo, en el que se contrastó la hipótesis de que el capital social influye de manera directa y positiva en la calidad de vida de la comunidad receptora. A efectos de contrastar empíricamente esta hipótesis, se diseñó un estudio de corte transversal mediante encuesta aplicada durante el verano de 2017 a residentes en la isla de Cozumel, y análisis mediante modelos de ecuaciones estructurales (SEM). Los resultados arrojaron relaciones directas y positivas de las dimensiones cognitiva y de habilidades sociales del capital social sobre los dominios de satisfacción con el bienestar emocional, comunitario, seguridad y salud y material, así como sobre la medida global de una vida satisfactoria. Se presentan los resultados detallados, su análisis y discusión, así como conclusiones, limitaciones y futuras líneas de investigación en esta temática.

Palabras clave: Destino turístico de sol y playa, Capital Social, Calidad de Vida, Cozumel

\section{ABSTRACT}

Living on an island which is a popular tourist destination, offering a mass sun and beach product, as is the case of Cozumel, Mexico, is not equivalent to living in paradise, as pointed 
out by the academic literature since it has been analysing the social, economic, cultural and environmental impacts of tourism on the destinations. There is empirical evidence to show that the perception of economic benefits derived from tourism has a positive impact on the formation of social capital in the community and also on the perception of an improvement in the quality of life, however there are not many studies that examine the relationship between these variables in depth. The central object of this study is to test the hypothesis that social capital directly and positively influences the quality of life of the receiving community. In order to empirically test this hypothesis, a cross-sectional study was designed through a survey which was applied during the summer of 2017 to residents on the island of Cozumel. The results were analyzed using structural equation models (SEM). The results revealed direct and positive relationships of the cognitive and social skills dimensions of social capital on the domains of satisfaction with emotional, community, safety and health and material well-being, as well as on the overall measure of life satisfaction. The detailed results, their analysis and discussion, together with the conclusions, limitations and future lines of research in this subject are presented.

Keywords: "Sun and beach" tourism destination, Social Capital, Quality of Life, Cozumel

\section{INTRODUCCIÓN}

La llegada de turistas y el crecimiento del sector económico turístico en los sitios de destino, incide de manera importante en el tejido social de la sociedad anfitriona (Deery et al., 2012; Sharpley, 2014). Por una parte la confrontación o afinidad que puede surgir entre sistemas de valores compartidos o no entre turistas y anfitriones se constituye como un factor de cambio en la sociedad anfitriona (Monterrubio, 2008; Woosnam et al., 2009) y por otra, las diferencias o asimetrías en la relación y aceptación de turistas entre distintos grupos de anfitriones hacen posible la generación de fracturas y conflictos internos en la sociedad receptora (Frauman y Banks, 2011; Sharma et al., 2008).

Cuando los impactos son percibidos como positivos por parte de la sociedad anfitriona, la sociedad en su conjunto muestra una mayor disposición a apoyar el desarrollo de la actividad turística (Choi y Murray, 2010; Rasoolimanesh et al., 2017), por ello, las administraciones locales en los sitios turísticos se interesan en encontrar mecanismos y estrategias que incentiven los impactos positivos y que atenúen los negativos (Fredline, 2002).

Se ha encontrado que las comunidades que reciben turismo son más o menos susceptibles a estos impactos, debido a factores preexistentes en ellas, tales como la desigualdad, el nivel de ingresos, las oportunidades de empleo, los indicadores de bienestar, la calidad de vida percibida y las condiciones del capital social de la sociedad anfitriona (Baker y Faulkner, 2009; González et al., 2009).

Los impactos del turismo en la sociedad han sido objeto de interés tanto en los ámbitos académico como práctico, dado que la actividad ha sido promovida como una alternativa para el desarrollo regional, para combatir la pobreza y para aprovechar de manera sostenible los recursos con los que cuentan poblaciones y territorios. No obstante, los estudios sobre los impactos se han centrado en gran medida en su identificación y su valoración tanto positiva 
como negativa para los sitios de destino turístico (Monterrubio, 2018), poco se ha profundizado en las formas en las que estos impactos se relacionan, retroalimentan y condicionan entre sí.

En este sentido, se planteó el objetivo de analizar las relaciones estructurales entre capital social y calidad de vida de la comunidad receptora en un destino turístico, como un par de indicadores de la salud de la sociedad, que por sí mismos han sido analizados en la sociedad en general y también lo han sido en particular como aspectos impactados por la presencia del turismo.

El capital social en un grupo, colectivo o comunidad es un indicador que hace referencia a la fortaleza de las relaciones de confianza, solidaridad y reciprocidad entre los individuos que los conforman (Portes, 1998; Kwon y Adler, 2014) y que les otorga la capacidad de actuar colectivamente en torno a objetivos o problemas que enfrenten (Ostrom y Ahn, 2009). Este capital se construye socialmente y si bien en gran medida depende de la voluntad de los individuos, es matizado por las costumbres, las creencias y la religión, las tradiciones, la ideología (Coleman, 1988). En términos generales está determinado por la cosmovisión que el propio colectivo históricamente ha conformado, aunque tiene carácter dinámico, se transforma permanentemente en cada relación entre los individuos (Lin, 2001). Asimismo, el capital social es susceptible de verse influenciado por situaciones contextuales, por acciones colectivas o individuales internas o externas al colectivo o grupo (Putnam, 1995). Esta susceptibilidad del capital social, ha generado interés por su análisis para el caso de las sociedades receptoras de turismo, en las que se supone de antemano un contacto entre individuos con cosmovisiones distintas, anfitriones y turistas.

A su vez, el bienestar percibido por los habitantes de una sociedad receptora se puede sintetizar en el concepto de calidad de vida, concepto que se ha desarrollado como un indicador de las condiciones materiales, emocionales, comunitarias y relacionales que los miembros de una sociedad perciben en su vida cotidiana. La calidad de vida se ha concebido teóricamente cuando se le observa desde la subjetividad individual, como una condición que combina en una percepción de satisfacción general con la vida, cinco dominios de satisfacción particulares: a) dominio del bienestar comunitario, que se refiere a las condiciones del entorno habitual de residencia, b) dominio del bienestar material, referido a las condiciones económicas y capacidad de la persona para adquirir satisfactores, c) dominio del bienestar medioambiental, que incluye las condiciones del entorno natural, d) dominio del bienestar emocional, que hace referencia a las condiciones y posibilidades de disfrutar en un sentido más hedónico del tiempo y las relaciones interpersonales, y e) dominio de la satisfacción con las condiciones de salud y seguridad, que alude tanto a la salud personal como a la seguridad en su entorno inmediato (Kim, et al., 2013).

El incremento del capital social de un grupo social o comunidad puede influir favorablemente en su percepción subjetiva de bienestar, en su calidad de vida, particularmente cuando se moviliza el capital social en favor de los objetivos comunes para los miembros del grupo (Chesney y Hazari, 2003; Kim y Kawachi, 2007; Nilsson, et al., 2006; Requena, 2003; Teye, et al., 2002). Si bien esta relación no ha sido explorada en detalle en el ámbito de los estudios del turismo, se considera que su análisis detallado puede resultar esclarecedor en tanto 
conocimiento de los impactos sociales del turismo, como útil en términos prácticos para ser tomados en cuenta como parte de los esfuerzos de planificación y desarrollo en los destinos turísticos (Soares y Gandara, 2012).

Para tal efecto, se diseñó un estudio empírico en Cozumel, Quintana Roo, México, sitio turístico del tipo denominado de sol y playa, con oferta especializada en el turismo de buceo y en el turismo de cruceros. En las siguientes líneas, se desarrollan los resultados de tal estudio, en primer lugar la fundamentación teórico-conceptual que condujo al planteamiento del modelo hipotético a ser contrastado empíricamente, a continuación el diseño metodológico que permitió conducir el estudio empírico, los resultados y su discusión, para finalmente plantearse conclusiones que de ellos se desprendieron, así como una reflexión sobre las limitaciones del estudio, las futuras líneas de investigación que se sugieren a partir de éste y posibles recomendaciones orientadas a la planificación y desarrollo de destinos turísticos.

\section{ANTECEDENTES}

\section{Capital social en las comunidades receptoras de turismo}

Con capital social se hace referencia a las redes de relaciones sociales de confianza y colaboración recíproca que se establecen entre miembros de una organización o grupo social que permiten su desarrollo institucional, comunitario, colectivo o grupal (Kwon y Adler, 2014). Es un concepto trabajado en el ámbito de las ciencias sociales desde mediados del siglo XX (Häuberer, 2011) y que si bien ha sido objeto de críticas y debate académico (Woolcock, 2010), se reconoce su valor como elemento fundamental de las sociedades y ha conseguido posicionarse como objeto teórico y empírico de permanente análisis y debate.

Paul S. Adler y Seok-Woo Kwon (2002 y 2014) en un esfuerzo de integración de una larga tradición de estudios del capital social desde perspectivas teóricas discrepantes entre sí (Bourdieu, 1980; Coleman y Coleman, 1994; Putnam, 1993), definen al capital social como la "buena voluntad hacia individuos y grupos, donde buena voluntad se refiere a un sentir o actitud amable, atenta o amigable" (Kwon y Adler, 2014: 412). En su propuesta teórica integradora, los autores, plantean por una parte que los efectos benéficos del capital social son observables en el aumento de información, influencia y solidaridad entre individuos (capital social vínculo) y entre actores individuales y colectivos (capital social puenteo). A su vez, plantean que las fuentes del capital social descansan en las relaciones sociales entre los actores y que tales relaciones tienen naturaleza distinta que aquellas que se desprenden de las vinculaciones de intercambio comercial y de las que se derivan de la autoridad jerárquica (Granovetter, 1992).

Kwon y Adler (2014), identifican tres dimensiones constituyentes del capital social que puede ser desarrollado, acumulado, entre los integrantes de una comunidad o grupo, a las que denominan en términos genéricos: motivación, oportunidad y habilidad (MOA) (Blumberg y Pringle, 1986). Los autores equiparan a lo que denominan capital social cognitivo con la dimensión de motivación, a la que nombran capital social estructural con la dimensión de oportunidad y a las habilidades sociales con una dimensión activa o de puesta en acción del capital social. Cabe señalar, que su propuesta teórica difiere de otras que también son usuales 
en los estudios sobre el capital social, en las que se utilizan diversas denominaciones para las distintas dimensiones del capital social.

No existe consenso general entre autores ni en el número ni en el concepto de cada una de las dimensiones del capital social, por citar algunos, Granovetter (1992) identificó dos dimensiones a las que denominó dimensión estructural embebida y dimensión relacional embebida; Nahapiet y Ghoshal $(1997,1998)$ identificaron tres, a las que denominaron estructural, relacional y cognitiva; Moran (2005) identificó dos: estructural y relacional; Uphoff (1999) mencionó dos: estructural y cognitiva, Casanueva et al. (2013) identificaron cinco: estructural, cohesión, diversidad, relacional y recursos; en tanto que diversos autores no las conciben como dimensiones sino como factores, como sucede con Coleman (1990) que menciona como tales, las obligaciones, expectativas y confianza, canales de información, normas y sanciones eficaces, relaciones de autoridad y término de relaciones o Putnam (1993) que los denomina características de una organización social, confianza, normas de reciprocidad y sistemas o redes de compromiso cívico. Si bien en el presente trabajo no se pretende debatir en cuanto a las dimensiones en sí, se tomaron las definiciones de las tres elegidas con base en la mencionada propuesta de Kwon y Adler (2014).

La dimensión estructural del capital social se refiere a las redes de relaciones que establecen los miembros de una comunidad entre ellos y con otros actores institucionales o individuales dentro o fuera de su espacio geográfico y social (Kwon y Adler, 2014). Estas redes de relaciones pueden ser vistas como una estructura de vínculos, que se ha conformado a través de la historia de los miembros de la comunidad, se refiere pues a la forma del capital social.

Por su parte, la dimensión cognitiva del capital social, se centra en la confianza, la cooperación y solidaridad que los miembros de la comunidad reconocen en sus relaciones entre sí, ubicada en un ámbito más próximo a lo individual y lo subjetivo para cada uno de los miembros de una colectividad (Adler y Kwon, 2002), es decir se refiere a las cualidades atribuidas a los vínculos por lo tanto se puede reconocer como el contenido en las relaciones en una comunidad. Otros autores separan el contenido de las relaciones en dos (García-Villaverde et al., 2017; Nahapiet y Ghoshal, 1997, 1998; Pearson et al., 2008; Tsai y Ghoshal, 1998), denominando dimensión cognitiva al conjunto de valores y discursos compartidos por los miembros del grupo, y dimensión relacional al contenido atribuido a la relación por los actores vinculados.

Siguiendo a Kwon y Adler (2014), plantean como tercera dimensión del capital social, a aquella constituida por las habilidades sociales, que son las que permiten a los actores, individuales o grupales, hacer uso de las redes de vínculos, para movilizar la confianza y la cooperación y por ende, alcanzar los beneficios de la acumulación del capital social.

En el ámbito del desarrollo turístico en las comunidades receptoras, el rol del capital social se ha encontrado, en sus distintas dimensiones, como central y condición relevante para el desarrollo local (Merinero, 2008; Veloso y Delgado, 2008), el desempeño grupal y organizacional (Dai et al., 2015; Li y Hunter, 2015; Ramírez, et al., 2018), en la innovación organizacional (García-Villaverde, et al., 2017; Martínez-Pérez et al., 2016), el turismo rural (González, et al., 2016; Park, et al., 2012), la acción colectiva (Sandoval y Ortega, 2018) y la participación cívica 
responsable (Ziakas y Costa, 2010) y para otras formas de capital (McGehee, et al., 2010). Se ha encontrado evidencia empírica de que el desarrollo del turismo como actividad económica central o complementaria en una comunidad, favorece la formación y acumulación de capital social entre los habitantes de los sitios receptores de turismo, trátese de espacios rurales (González, et al., 2016; Liu et al., 2014) o urbanos (González y Herrera, 2017; Merinero, 2008; Oliveira y Pimentel, 2016). En tal sentido, se puede afirmar que la presencia de la actividad turística influye en las condiciones del capital social de la comunidad, por lo que su cambio a lo largo del tiempo puede a su vez ser relacionado con otros aspectos de la sociedad que también son modificados por la presencia del turismo, uno de ellos, el que interesa en el presente trabajo es la calidad de vida de la población residente en la comunidad receptora.

\section{La calidad de vida y el capital social en destinos turísticos}

Si bien el tema de la calidad de vida en los sitios turísticos ha cobrado interés en el ámbito académico en tiempos relativamente recientes (Uysal, et al., 2012; Yu, et al., 2016), algunos de sus elementos mantienen presencia en trabajos elaborados desde la década de los 80 del siglo XX, sin que necesariamente a ello se le hubiera llamado calidad de vida en la acepción que actualmente se le otorga (Uysal et al., 2012). Entre estos aspectos se encuentran por ejemplo, las cualidades socioeconómicas del turismo percibidas como beneficiosas para la sociedad anfitriona (Andereck y Nyaupane, 2011; Perez y Camberos, 2017), el enriquecimiento de la vida como efecto indirecto de la inversión, que se realiza para atraer turistas, en los sitios de destino (Castillo, et al., 2015) e incluso en términos del análisis de los indicadores objetivos de bienestar de los sitios con impactos por actividad turística a lo largo de su ciclo de vida (Faulkner, y Tideswell, 1997; Fredline, 2002; Sharma et al., 2008)

En tiempos recientes, el interés en general por entender los factores que constituyen lo que subjetivamente las personas entienden como calidad de vida, ha dado pie al surgimiento de grupos internacionales que se enfocan en su estudio sistemático. Por una parte los dedicados a su evaluación empírica y a la difusión de los resultados con fines comparativos (Land, et. al., 2011, Sirgy, 2012). Por otra, los enfocados en la discusión conceptual con orientación crítica de la noción misma de calidad de vida vinculada y a veces encontrada, con otros conceptos tales como los de bienestar, buen vivir o felicidad (Camfield y Skevington, 2008).

Se puede afirmar que tales trabajos han llevado a un cierto nivel de acuerdo en algunos de los elementos centrales del concepto de calidad de vida: a) la calidad de vida de un grupo o colectividad puede ser analizada, estudiada desde perspectivas objetivas, subjetivas o mixtas (Moscardo, 2009), b) se ubica en un ámbito de la sociedad humana que trasciende a los indicadores puramente económicos como el ingreso o la desigualdad (Land et al., 2011), c) en el concepto de calidad de vida se reconoce la búsqueda eudaimónica de una vida plena, más que la persecución hedónica de satisfactores placenteros inmediatos (Nawijn y Filep, 2016) y d) la calidad de vida desde el ámbito subjetivo personal e interpersonal es tan o más importante que los indicadores objetivos o socioeconómicos globales o agregados (McCabe y Johnson, 2013). 
El interés en lo general en la temática de la calidad de vida se ha reflejado también en un incremento en el número de estudios que la abordan en su relación con el turismo (Nawijn y Filep, 2016; Tang, 2014; Uysal et al., 2016). Cuando se relaciona al turismo con la calidad de vida, se le ha vinculado a los impactos de la presencia y desarrollo de la actividad (Butler, 1980; Doxey, 1975); se ha relacionado con los efectos de la visita a familiares y amigos (González y Macías, 2017) y con el apoyo de la comunidad al desarrollo del turismo (Nunkoo y Gursoy, 2012; Sharpley, 2014; Woo, et al., 2015). Particular atención se ha puesto en el hecho de que el desarrollo del turismo se ve frenado cuando la calidad de vida se percibe afectada negativamente por el crecimiento intenso y disruptivo de la vida cotidiana local (Ap y Cropmton, 1993, 1998; Doğan, 1989; Urtasun y Gutiérrez, 2006). Asimismo, hay estudios específicos sobre la calidad de vida de la fuerza laboral del sector turismo (Kay y Diekmann, 2017; Zoghbi, et. al., 2016).

La calidad de vida subjetivamente percibida por los habitantes de un sitio turístico, es influenciada por diversos factores internos y externos a la persona. Entre ellos, puede destacarse la relevancia que tienen las relaciones e interacciones con otras personas en la formación de la percepción subjetiva de la calidad de vida, la satisfacción con la vida en términos globales y los distintos dominios del bienestar (Sirgy, 2017).

Las relaciones entre las personas que integran una comunidad, que dan forma y contenido a su capital social, son influenciadas por la presencia de la actividad turística de manera diferenciable, a lo largo del ciclo de vida del sitio turístico (Butler, 1980), tanto en el nivel individual, propiciando encuentros interpersonales con personas con orígenes culturales distintos a los propios (García y González, 2018, Teye et al., 2002) como en un nivel social, al establecerse nuevas estructuras y organizaciones sociales para atender al turista y gestionar en general la actividad y sus impactos (Yu et al., 2016). Estos cambios propiciados o incentivados por la actividad turística en la estructura social a su vez tiene un impacto en la percepción de la calidad de vida de los residentes en el sitio turístico, por lo que se puede afirmar que, las distintas dimensiones en las que opera el capital social de los habitantes del sitio turístico, influyen en la calidad de vida, tanto en los dominios del bienestar como en la percepción global de la satisfacción con la vida.

\subsection{Capital social estructural y los dominios de la vida}

En la dimensión de oportunidad o capital social estructural, las relaciones y lazos que se forman entre los miembros de una comunidad influyen en la calidad de vidad de los habitantes, específicamente lo hacen respecto a la percepción de que existe una buena o satisfactoria interacción con los vecinos y en general con la gente que vive en la comunidad (Bruin y Cook, 1997; Sirgy y Cornwell, 2002).

Las oportunidades que tienen las personas en una comunidad que recibe turismo de establecer relaciones de confianza duraderas y mutuamente beneficiosas, que se traducen en más y mejores relaciones con otros actores, le confieren una percepción de identidad y pertenencia al grupo (Kawachi y Berkman, 2000; Lochner et al., 2003; Younsi y Chakroun, 2016). La densidad y fortaleza del conjunto de relaciones interpersonales promueve el equilibrio 
emocional de las personas, si bien esta relación se ha señalado desde hace largo tiempo en la literatura (Durkheim, 1971) de manera más clara ante la ausencia de buenas relaciones interpersonales. La ausencia de relaciones o vínculos con otros miembros de la comunidad empeora las condiciones precarias de salud en tanto que su efecto es menor para la mejoría de la salud cuando hay alto número de relaciones y vínculos sociales (Inaba, et al., 2015; Hausman, et al., 2005; Kim y Kawachi, 2007; Lochner, et al., 2003; Maas, et al., 2016; Younsi y Chakroun, 2016).

La existencia de redes de densas, en las que existe alta relación entre los miembros de una comunidad, las personas encuentran con mayor probabilidad canales de comunicación y de proveeduría de cuidados, apoyo, respaldo, confort, de manera más rápida o directa en los demás miembros de su comunidad (Carpiano, 2007; Elgar, et al., 2011; Vemuri, et al., 2011; Subramanian, et al., 2002; Wen, et al., 2003; Younsi y Chakroun, 2016).

La existencia de las redes de vínculos, especialmente cuando se consideran duraderas, influyen en la percepción de que al existir organizaciones y estructuras centradas en la actividad económica, como lo es la turística, contribuyen directa e indirectamente a una mejoría en las condiciones materiales y del entorno (Kawachi y Berkman, 2000), lo que a su vez propicia una percepción de mayor seguridad y tranquilidad (Hamdan, et al., 2014; Pitchford, 2008) entre los miembros de la comunidad, una tendencia mayor a evitar el daño ambiental, a cuidar el ambiente de trabajo, a disminuir las prácticas negativas para la salud (Younsi y Chakroun, 2016). Con base en estos argumentos, se plantea el primer conjunto de hipótesis.

Hipótesis 1: El capital social estructural influye de manera directa y positiva en el dominio de la satisfacción con ...

... el bienestar comunitario ( $\mathrm{H} 1 \mathrm{a})$

... el bienestar emocional ( $\mathrm{H} 1 \mathrm{~b})$

... el bienestar material (H1C)

... el bienestar medioambiental (H1d)

... la seguridad y salud (H1e)

\subsection{Capital social cognitivo y los dominios de la vida}

En la dimensión de motivación o capital social cognitivo, la confianza, confiabilidad, respeto, identificación, cooperación, solidaridad y en general los valores que las personas atribuyen a sus relaciones con los demás miembros de su comunidad, influye en la satisfacción con la vida en general, por lo que promover el capital social en una comunidad puede ser una estrategia beneficiosa para promoverla (Diener et al., 2009; Maass, et al., 2016; Rablen, 2012).

De hecho, se ha encontrado que mientras menor sea la confianza entre los ciudadanos, se observan incrementos en las tasas de mortandad (Berkman y Syme 1979; Kawachi y Berkman, 2000), aunque esta relación es muy posible que se encuentre mediada por la presencia de otro conjunto de variables, tales como el estrato socioeconómico (Aminzadeth, et al., 2013), la violencia, la delincuencia, e incluso la guerra (Inaba, et al., 2015).

En la medida en la que las relaciones con los demás miembros de la comunidad son valoradas favorablemente, en términos de confianza, cooperación, identificación, solidaridad, 
los miembros de una comunidad encuentran más satisfactoria tal convivencia con las demás personas que viven en su entorno (Lyubomirsky, et al., 2005; Vrbka y Combs, 1993; Sirgy y Cornwell, 2002), esto es, que muestra un efecto directo en el bienestar comunitario y en la percepción de su entorno inmediato (Eriksson, 2011; Vemuri, et al., 2011) y facilita la cooperación que a su vez da acceso al apoyo, ayuda y servicios provistos por instituciones formales e informales, basadas en la reciprocidad (Younsi y Chakroun, 2016). Estos atributos de las relaciones, influyen directamente en las posibilidades que las personas perciben que tiene de jugar y realizar actividades en el exterior o en general en la comunidad sin ser molestadas y a comportamientos saludables (Mohnen, et al., 2012), la percepción de peligrosidad o criminalidad del entorno (Galster y Hesser, 1981; Sirgy y Cornwell, 2002) y la percepción de privacidad en de su entorno inmediato (Bruin y Cook, 1997; Sirgy y Cornwell, 2002).

Los motivos que favorecen el establecimiento de redes de solidaridad y colaboración entre los miembros de una comunidad que recibe turismo tales como la confianza en los demás, el valorar los objetivos colectivos por arriba de los individuales, la solidaridad, se vinculan con valores personales e interpersonales que favorecen la autoestima y un sentido de coherencia (Briggs, 2010; Maass, et al., 2016; Wiesmann y Hannich, 2013) que fortalecen la disposición de las personas a colaborar entre sí, de modo que tiene la posibilidad de influir en la percepción de una vida satisfactoria en sus cinco dominios. En conjunto, estos argumentos permiten plantear el segundo conjunto de hipótesis.

Hipótesis 2: El capital social cognitivo influye de manera directa y positiva en los dominios de la satisfacción con ...

... el bienestar comunitario ( $\mathrm{H} 2 \mathrm{a})$

... el bienestar emocional $(\mathrm{H} 2 \mathrm{~b})$

... el bienestar material ( $\mathrm{H} 2 \mathrm{C})$

... el bienestar medioambiental (H2d)

... la seguridad y salud (H2e)

\subsection{Habilidades sociales y los dominios de la vida}

En la dimensión de habilidades como dimensión del capital social, se encuentran las habilidades y aptitudes desarrolladas por los miembros de una comunidad para aprovechar las relaciones y lazos que han conseguido crear con los otros miembros de la comunidad. El contar con estas habilidades en mayor medida, también contribuye favorablemente en los distintos dominios del bienestar vital de las personas, no únicamente por contar con una percepción de la capacidad de actuar en colectivo, sino también el percibir que en caso de ser necesario ante alguna eventualidad, la persona cuenta con la capacidad de movilizar recursos colectivos ya sea para ayudar a otros miembros de la comunidad o a sí misma. En este sentido, las habilidades sociales influyen en la satisfacción de contar con lazos con la gente que habita en la misma comunidad (Sirgy y Cornwell, 2002), en la percepción de una capacidad de actuar contra disturbios del entorno como pueden ser los niveles de ruido o de aglomeramiento (Bonnes, et al., 1991; Sirgy y Cornwell, 2002), en la percepción de que se tiene la capacidad de actuar en concordancia con el estatus socioeconómico del vecindario y en colaboración y solidaridad con la gente que vive en la comunidad (Lu, 1999, Sirgy y Cornwell, 2002). 
Si bien, la dimensión de las habilidades sociales, no ha sido considerada en el estudio del capital social de manera extensa en la literatura, la propuesta de su inclusión (Adler y Kwon, 2002) tiene posibilidades de aportar una mejor comprensión de sus relaciones con otros aspectos de la vida social, como lo es el de la calidad de vida, lo cual permite postular el tercer conjunto de hipótesis. Las habilidades que los miembros de una comunidad han desarrollado para relacionarse entre sí y para movilizar en beneficio colectivo tales relaciones forma parte del capital social de la comunidad, en particular, la habilidad social para percibir y reconocer situaciones colectivas en las que la persona regula su forma de participación ante la misma (Allen y Gibson, 2009) tiene la posibilidad de influir en la satisfacción con la vida en sus cinco dominios.

Hipótesis 3: La habilidad para la percepción social de la persona influye de manera directa y positiva en los dominios de la satisfacción con ...

... el bienestar comunitario ( $\mathrm{H} 3 \mathrm{a}$ )

... el bienestar emocional (H3b)

... el bienestar material ( $\mathrm{H} 3 \mathrm{C})$

... el bienestar medioambiental (H3d)

... la seguridad y salud (H3e)

El segundo conjunto de habilidades sociales relevante en el ámbito de la formación y uso del capital social en las comunidades, lo constituye la de integrarse, ajustarse a las situaciones sociales para participar en actividades de beneficio colectivo (Gabbay y Leenders, 1999; Lin, 1999), habilidad que a su vez también tiene la posibilidad de influir en la satisfacción con la vida en sus cinco dominios, lo que permite postular el cuarto conjunto de hipótesis.

Hipótesis 4: La habilidad para el ajuste social de la persona influye de manera directa y positiva en los dominios de la satisfacción con ...

... el bienestar comunitario ( $\mathrm{H} 4 \mathrm{a}$ )

... el bienestar emocional ( $\mathrm{H} 4 \mathrm{~b})$

... el bienestar material ( $\mathrm{H} 4 \mathrm{C})$

... el bienestar medioambiental (H4d)

... la seguridad y salud ( $\mathrm{H} 4 \mathrm{e})$

\subsection{Los dominios de la vida y la satisfacción global con la vida}

La calidad de vida como percepción subjetiva es una condición compleja, que ha sido abordada para su estudio como constructo compuesto, conformado por las relaciones de tipo formativo entre el bienestar con los dominios de la vida de las personas y su satisfacción general o globalmente percibida con la vida (Diener, et al., 1999; Sirgy, et al., 2000). Esta forma de concebir la calidad de vida parte del supuesto de que existe un conjunto de valores positivos que el ser humano persigue a través de su actuar, para alcanzar una condición de bienestar, felicidad o eudaimonía y que tales valores inciden directamente en su satisfacción.

Entendida de este modo, la satisfacción global con la vida se encontraría en la cima de la satisfacción subjetiva con la calidad de vida, siendo influida por la satisfacción con el bienestar en los distintos dominios de la vida, a su vez la satisfacción en cada dominio particular es 
influenciado por niveles menores o sub-dominios pertinentes con ese dominio. Así, mientras mayor es la satisfacción con el bienestar en cada uno de los dominios principales de la vida, mayor es la satisfacción general y por ende, se puede concebir como una mejor calidad de vida percibida subjetivamente, en un entrelazamiento denominado teoría del desbordamiento de abajo hacia arriba (Bottom-up spillover theory) y utilizado ampliamente en los estudios de calidad de vida (Sirgy, et al., 2000).

Cada uno de los dominios de la satisfacción, entonces son a su vez una función de distintos subdominios, originalmente planteados en términos de niveles, desde el nivel global o general, pasando por los niveles estatal, comunitario, grupal e individual (Sirgy y Cornwell, 2001). Si bien, en su forma original, el modelo planteado de esta manera hace suponer relaciones formativas de los aspectos individualmente observados, agrupados por sumatoria o promedios ponderados, en otros trabajos, el modelo se ha revisado concibiendo a cada dominio del bienestar como variable latente reflectiva, accesible de manera indirecta a través de los aspectos individuales o ítems evaluados (Fayers y Hand, 1997; Lee, 2008; Lee, et al., 2005).

La calidad de vida, en tal sentido, se forma como constructo o variable latente, en la relación de los dominios de la satisfacción con la vida y la percepción global de gozar de una vida satisfactoria, en el caso de las comunidades que reciben turismo, esto opera también desde los dominios de satisfacción comunitaria, medioambiental, emocional, material y de condiciones de salud y seguridad en conjunto.

Hipótesis 5 a 9: La satisfacción global con la vida que manifiestan las personas es influida de manera directa y positiva por ...

... el bienestar comunitario (H5)

... el bienestar emocional (H6)

... el bienestar material (H7)

... el bienestar medioambiental (H8)

... la seguridad y salud (H9)

Estas relaciones teóricamente planteadas y que han sido probadas empíricamente en ámbitos no necesariamente turísticos, permite dar sustento al modelo hipotético a ser contrastado empíricamente, el cual se describe a continuación y puede ser observado esquemáticamente en la figura 1. 
Figura 1. Modelo hipotético

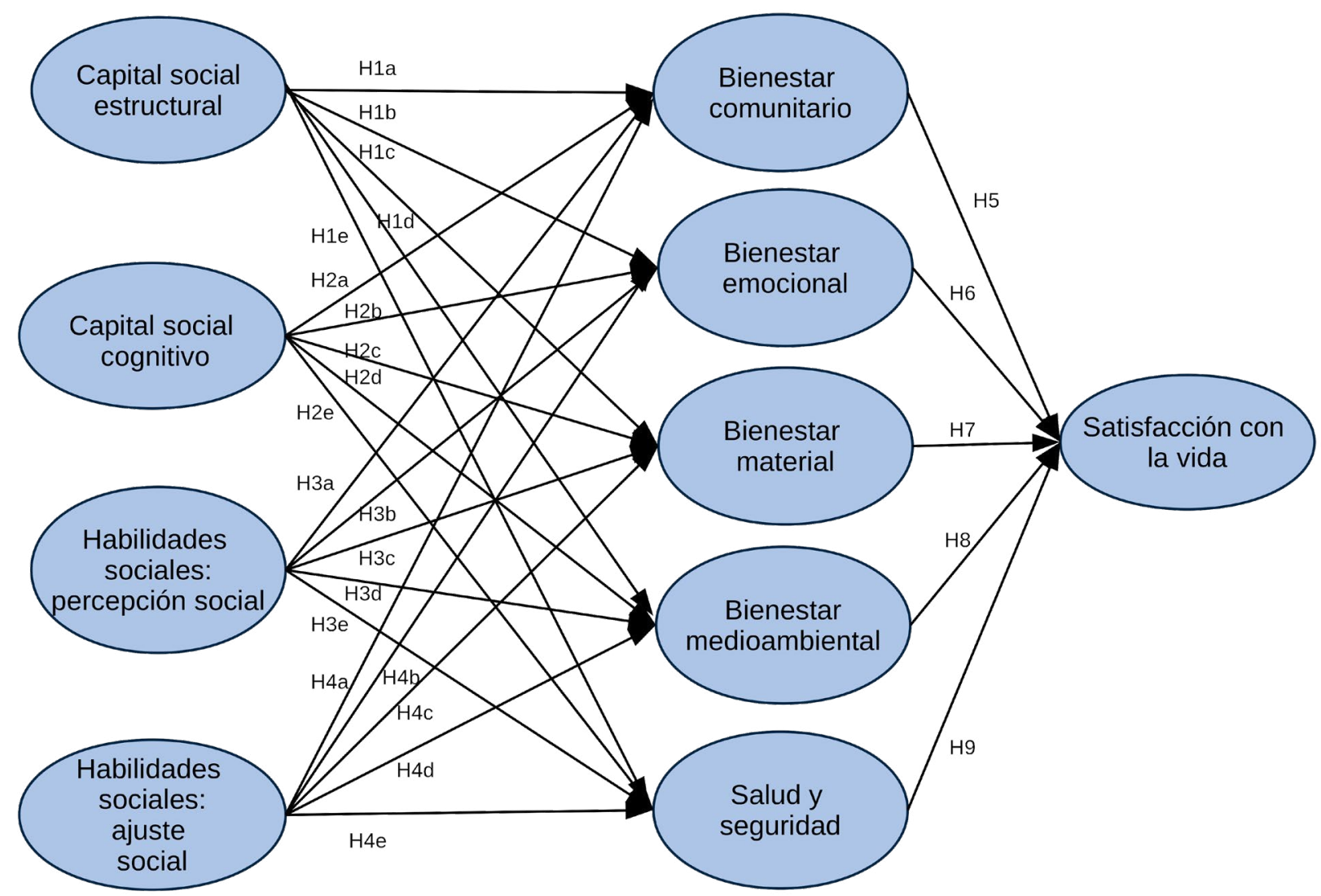

Fuente: elaboración propia.

\section{METOdOLOGÍA Y ÁMBITO DEL ESTUDIO}

\section{1. Ámbito del estudio}

El estudio se realizó en Cozumel, México, isla turística con oferta de sol y playa, especializada en atención de turismo de cruceros y de turismo de buceo, se ubica en el extremo noroeste de la península de Yucatán en el mar Caribe. En Cozumel se ha instalado la industria turística para posicionarse como la principal actividad económica para los cerca de 80 mil habitantes con los que cuenta actualmente (INEGI, 2010) y recibir a casi 5 millones de visitantes extranjeros al año (Secretaría de Desarrollo Turístico del Estado de Quintana Roo, 2017) así como un número estimado de 3 millones de visitantes nacionales. Esta actividad que ha crecido anualmente desde hace 30 años, constituye una presión permanente que ha impactado y lo sigue haciendo económica, social, cultural y ambientalmente al destino y por ende a la comunidad residente en la isla, que se asienta principalmente en la ciudad de Cozumel, con una proporción menor en la comunidad de El Cedral y otras en un territorio de $478 \mathrm{~km}^{2}$ de extensión. Cabe destacar que las zonas costeras en un alto porcentaje y parte del territorio interno en la isla se ha decretado como área natural protegida: Parque nacional Arrecifes de Cozumel, Laguna Colombia y Selvas y humedales de Cozumel (SECTUR, Gobierno del Estado de Quintana Roo, y Universidad de Quintana Roo, 2014). 


\section{Diseño metodológico}

Se realizó una aproximación empírica con el fin de evaluar el modelo hipotético postulado en el apartado anterior, para lo cual se seleccionó un sitio de destino turístico convencional con oferta de sol y playa, en el que se realizó un estudio de corte transversal, basado en encuesta aplicada en hogares, a una muestra de personas residentes en ocho colonias de un total de 23 que existen en Cozumel, registradas en datos del Instituto Nacional de Estadística y Geografía (INEGI, 2015) ${ }^{1}$. Las colonias fueron seleccionadas aleatoriamente y en ellas se aplicaron cuotas de 50 cuestionarios, durante los meses de junio a septiembre de 2017. Los cuestionarios se integraron por tres conjuntos de preguntas (ítems), el primero de ellos para evaluar la calidad de vida percibida por los residentes, el segundo para evaluar el capital social de los residentes y el tercero para obtener una descripción del perfil sociodemográfico de los encuestados.

La muestra finalmente integrada por 266 observaciones válidas, conformó la base de datos sobre la que se realizaron, primero un análisis factorial confirmatorio (CFA) para evaluar la validez y fiabilidad compuesta de las escalas utilizadas, las cuales se describen más adelante en este mismo apartado, y posteriormente se ajustó un modelo de ecuaciones estructurales mediante análisis de la estructura de covarianzas (Aldás Manzano y Uriel Jiménez, 2017) para contrastar empíricamente de manera simultánea las nueve hipótesis postuladas. Para la realización de ambos análisis se hizo uso del lenguaje de programación para análisis estadístico $\mathrm{R}$ (R Core Team, 2018), específicamente con el paquete lavaan (Rosseel, 2012).

\section{Métrica de las variables de interés}

Las variables, capital social y calidad de vida fueron evaluadas mediante la aplicación de escalas compuestas, para variables latentes o también llamados constructos, no observables directamente. Ambas se constituyen por un conjunto de ítems que se consideran indicadores de la variable latente cuando se evalúan en conjunto, cada uno de ellos medido en una escala de Likert de 5 puntos. El capital social fue evaluado mediante 13 ítems y la calidad de vida con 14 ítems, los cuales se muestran detalladamente a continuación en la tabla 1.

1. El número de colonias en Cozumel se ha incrementado en los años recientes, aunque el dato oficial más recientemente publicado indica que son 23, se estima que han llegado a cerca de 40 para el 2018 dado el crecimiento urbano observado. Para el presente estudio se eligieron 6 de las registradas y 2 más entre las no registradas oficialmente. 
Tabla 1. Escalas compuestas utilizadas en el estudio

\begin{tabular}{|c|c|}
\hline \multicolumn{2}{|r|}{ Variable: Capital social } \\
\hline Código & Ítem \\
\hline \multicolumn{2}{|c|}{ Dimensión: Capital social cognitivo (CSC, 3 ítems) } \\
\hline $\operatorname{Csc} 2$ & En mi comunidad los conflictos son poco frecuentes \\
\hline Csc3 & Las personas en mi comunidad son honestas \\
\hline Csc4 & Las personas en mi comunidad son confiables \\
\hline \multicolumn{2}{|c|}{ Dimensión: Capital social estructural (CSE, 4 ítems) } \\
\hline Cse1 & Mi participación en trabajos de beneficio comunitario es muy alta \\
\hline Cse2 & Frecuentemente participo como voluntario en mi comunidad \\
\hline Cse3 & Colaboro frecuentemente con al menos una organización de mi comunidad \\
\hline Cse4 & Participo en más de una organización en mi comunidad \\
\hline \multicolumn{2}{|c|}{ Dimensión: Habilidades sociales respecto a la percepción social (CSAPS, 3 ítems) } \\
\hline Csnps2 & $\begin{array}{l}\text { Usualmente puedo reconocer si me intentan engañar observando el comportamiento de las } \\
\text { personas }\end{array}$ \\
\hline Csnps3 & Usualmente puedo decir cómo se sienten los demás ante una situación dada \\
\hline Csnps4 & Generalmente soy capaz de entender por qué actúan las personas como lo hacen \\
\hline \multicolumn{2}{|c|}{ Dimensión: Habilidades sociales para el ajuste social (CSAAS, 3 ítems) } \\
\hline Csnas1 & Me ajusto fácilmente a casi cualquier situación social \\
\hline Csnas2 & Puedo sentirme cómodo al relacionarme con cualquier tipo de persona \\
\hline Csnas3 & Soy capaz de conversar con toda persona sin importar el tema de que se trate \\
\hline \multicolumn{2}{|r|}{ Variable: Calidad de Vida } \\
\hline \multicolumn{2}{|c|}{ Dominio de la satisfacción con seguridad y salud (SS, 2 ítems) } \\
\hline Ss $2 b$ & La familia \\
\hline Ss4b & Los amigos \\
\hline \multicolumn{2}{|c|}{ Dominio de la satisfacción con bienestar material (BM, 3 ítems) } \\
\hline $\mathrm{Bm} 1 \mathrm{~b}$ & Las compras \\
\hline $\mathrm{Bm} 2 \mathrm{~b}$ & La oportunidad de trabajo \\
\hline $\mathrm{Bm} 3 \mathrm{~b}$ & El costo de la vida \\
\hline \multicolumn{2}{|c|}{ Dominio de la satisfacción con bienestar emocional (BE, 2 ítems) } \\
\hline Be1b & Sus viajes de turismo \\
\hline $\mathrm{Be} 2 \mathrm{~b}$ & Las vacaciones \\
\hline \multicolumn{2}{|c|}{ Dominio de la satisfacción con bienestar medioambiental (BMA, 2 ítems) } \\
\hline Bma1b & El agua \\
\hline $\mathrm{Bma} 2 \mathrm{~b}$ & El aire \\
\hline \multicolumn{2}{|c|}{ Dominio de la satisfacción con bienestar comunitario (BC, 3 ítems) } \\
\hline $\mathrm{Bc} 1 \mathrm{~b}$ & El transporte \\
\hline $\mathrm{Bc} 2 \mathrm{~b}$ & Los servicios públicos \\
\hline $\mathrm{Bc} 3 \mathrm{~b}$ & Sus vecinos \\
\hline \multicolumn{2}{|c|}{ Satisfacción global con la vida (VS, 2 ítems) } \\
\hline $\begin{array}{l}\text { Vs1vc1 } \\
\text { Ss3b }\end{array}$ & $\begin{array}{l}\text { Con la vida en general } \\
\text { Con la vivienda }\end{array}$ \\
\hline
\end{tabular}

Fuente: elaboración propia con base en adaptación de las escalas desarrolladas por: a) capital social cognitivo y estructural, Liu, Qu y Huang (2014); habilidades sociales, Baron y Markman (2003) y calidad de vida, Kim, Uysal y Sirgy (2013). 
Con la finalidad de validar las escalas utilizadas, se efectuó un análisis factorial confirmatorio (CFA) para la escala de capital social y otro más para la escala de calidad de vida, los resultados se observan en las tablas 2 y 3 . Para el caso del capital social, en la tabla 2 , se ajustó un modelo mediante CFA y se obtuvieron valores aceptables de acuerdo con la literatura especializada (Aldás y Uriel, 2017), superiores a 0.9 en los indicadores TLI y CFI, así como un valor en el intervalo 0.05-0.1 para RMSEA. Con este modelo se analizaron los valores obtenidos para la medición de las variables latentes, capital social cognitivo (CSC), capital social estructural (CSE), percepción social (CSAPS) y ajuste social (CSAAS), cuyos resultados confirman la fiabilidad compuesta para cada una de ellas al arrojar valores para alpha de Cronbach y omega de McDonald superiores al umbral de 0.7 recomendado en estudios similares al presente y validez convergente con AVE superior a 0.5 .

Para el caso de la calidad de vida en la tabla 3, se efectuó también un CFA ajustando un modelo con valores aceptables, según la literatura (Aldás y Uriel, 2017), superiores a 0.9 en los indicadores TLI y CFI, así como un valor en el intervalo 0.05-0.1 para RMSEA, para este caso de 0.7.

Tabla 2. Fiabilidad compuesta y validez convergente de la escala de Capital Social

\begin{tabular}{|c|c|c|c|c|c|c|}
\hline Factor & Indicador & Lambda & $\mathbf{t}$ & $\alpha$ & $\omega$ & AVE \\
\hline \multirow[t]{3}{*}{$\operatorname{CSC}$} & Csc2 & $0.670^{*}$ & 12.049 & 0.8786653 & 0.8884630 & 0.7309260 \\
\hline & $\operatorname{Csc} 3$ & $0.942 *$ & 19.435 & & & \\
\hline & Csc4 & $0.924^{*}$ & 18.830 & & & \\
\hline \multirow[t]{4}{*}{ CSE } & Cse1 & $0.861^{*}$ & 17.453 & 0.9436986 & 0.9460911 & 0.8119178 \\
\hline & Cse2 & $0.963^{*}$ & 21.173 & & & \\
\hline & Cse3 & $0.919^{*}$ & 19.465 & & & \\
\hline & Cse4 & $0.856^{*}$ & 17.304 & & & \\
\hline \multirow[t]{3}{*}{ CSAPS } & Csnps2 & $0.621^{*}$ & 10.435 & 0.8060430 & 0.8071416 & 0.5947582 \\
\hline & Csnps3 & $0.794^{*}$ & 14.088 & & & \\
\hline & Csnps4 & $0.869 *$ & 15.804 & & & \\
\hline \multirow[t]{3}{*}{ CSAAS } & Csnas1 & $0.855^{*}$ & 16.901 & 0.9070669 & 0.9089912 & 0.7688716 \\
\hline & Csnas2 & $0.902^{*}$ & 18.359 & & & \\
\hline & Csnas3 & $0.869 *$ & 17.300 & & & \\
\hline
\end{tabular}

$\chi^{2}(75)=171.532 * ; \mathrm{CFI}=0.944 ; \mathrm{TLI}=0.921 ; \operatorname{RMSEA}(90 \%)=0.07(0.056 ; 0.083)$

$* \mathrm{p}<0.01$

Fuente: Elaboración propia 
Tabla 3. Validez convergente, fiabilidad compuesta de la escala de Calidad de Vida

\begin{tabular}{|c|c|c|c|c|c|c|}
\hline Factor & Indicador & Lambda & $\mathbf{t}$ & $\alpha$ & $\omega$ & AVE \\
\hline \multirow[t]{2}{*}{ SS } & $\mathrm{Ss} 2 \mathrm{~b}$ & $0.714^{*}$ & 10.248 & 0.6618828 & 0.6935158 & 0.5419177 \\
\hline & Ss4b & $0.746^{*}$ & 10.597 & & & \\
\hline \multirow[t]{3}{*}{$\mathrm{BM}$} & $\mathrm{Bm} 3 \mathrm{~b}$ & $0.641^{*}$ & 10.837 & 0.7956830 & 0.7852294 & 0.5617854 \\
\hline & $\mathrm{Bm} 2 \mathrm{~b}$ & $0.792^{*}$ & 14.272 & & & \\
\hline & $\mathrm{Bm} 1 \mathrm{~b}$ & $0.815^{*}$ & 14.84 & & & \\
\hline \multirow[t]{2}{*}{$\mathrm{BE}$} & Be1b & $0.912 *$ & 17.506 & 0.8823606 & 0.8823616 & 0.7894868 \\
\hline & $\mathrm{Be} 2 \mathrm{~b}$ & $0.867^{*}$ & 16.338 & & & \\
\hline \multirow[t]{2}{*}{ BMA } & Bma1b & $0.862 *$ & 15.329 & 0.8756337 & 0.8758415 & 0.7791499 \\
\hline & Bma2b & $0.906^{*}$ & 16.282 & & & \\
\hline \multirow[t]{3}{*}{$\mathrm{BC}$} & Bc1b & $0.768^{*}$ & 13.304 & 0.7932737 & 0.7973580 & 0.5649904 \\
\hline & $\mathrm{Bc} 2 \mathrm{~b}$ & $0.802 *$ & 14.036 & & & \\
\hline & $\mathrm{Bc} 3 \mathrm{~b}$ & $0.697^{*}$ & 11.819 & & & \\
\hline \multirow[t]{2}{*}{ VS } & Vs1vc1 & $0.590^{*}$ & 9.118 & 0.6264756 & 0.6675209 & 0.5176309 \\
\hline & Ss3b & $0.788^{*}$ & 11.738 & & & \\
\hline
\end{tabular}

$\chi^{2}(75)=171.532^{*} ; \mathrm{CFI}=0.944 ; \mathrm{TLI}=0.921 ; \operatorname{RMSEA}(90 \%)=0.070(0.056 ; 0.083)$

$* \mathrm{p}<0.01$

Fuente: Elaboración propia

En las variables constitutivas de la calidad de vida: bienestar material (BM), bienestar emocional (BE), bienestar medioambiental (BMA) y bienestar comunitario (BC) se encontraron valores de alpha, omega y AVE superiores al umbral recomendado de $0.7,0.7$ y 0.5 respectivamente. Por su parte las variables salud y seguridad (SS) y vida satisfactoria (VS) obtuvieron valores apenas por abajo de los umbrales recomendados por regla de oro (Taber, 2017), aunque al encontrarse en rangos moderadamente satisfactorios o aceptables, se optó por considerarlos útiles para el análisis, como se indica en diversos trabajos (Bojórquez et al., 2013; Cortina, 1993; Taber, 2017; van Griethuijsen et al., 2015), por lo que se puede afirmar que se confirman también su validez convergente y fiabilidad compuesta. Con estos resultados sobre la validez de las escalas, en las que particularmente se obtuvieron valores de lambda altos, se procedió con la evaluación del modelo hipotético.

\section{ANÁLISIS Y DISCUSIÓN DE RESULTADOS}

\section{Perfil sociodemográfico de los encuestados y descriptivos de las variables de interés}

En la tabla 4 se presenta de manera sintética el perfil sociodemográfico de los encuestados. Al respecto se puede destacar que los valores porcentuales para edad, sexo, lugar de nacimiento, escolaridad y años residiendo en Cozumel, son consistentes con los valores observados para la generalidad de la población: principalmente joven, alrededor de la mitad inmigrados no nacidos en la isla y con bajo nivel de estudios, de los que cerca de un tercio trabaja en actividades relacionadas con el turismo (INEGI, 2018).

El hecho de que las características de la muestra describan también a la población total de la isla de Cozumel, permite hacer inferencias respecto a los resultados que se obtuvieron 
del análisis, de la relación entre capital social y calidad de vida, las cuales se presentan en el siguiente apartado.

Tabla 4. Perfil sociodemográfico de los encuestados

\begin{tabular}{|c|c|c|c|c|c|}
\hline Edad & $\%$ & Lugar de nacimiento & $\%$ & Escolaridad & $\%$ \\
\hline 18-29 años & 35.71 & Cozumel & 49.25 & Licenciatura & 18.05 \\
\hline 30-39 años & 29.33 & Otra población de Quintana Roo & 11.65 & Posgrado & 0.38 \\
\hline 40-49 años & 22.93 & Total, Quintana Roo & 60.8 & Preparatoria & 34.21 \\
\hline 50-59 años & 9.77 & Yucatan & 24.81 & Primaria completa & 10.53 \\
\hline \multirow[t]{3}{*}{60 o más } & 2.26 & Campeche & 3.38 & Primaria incompleta & 3.01 \\
\hline & & Chiapas & 1.88 & Secundaria & 25.56 \\
\hline & & Otro estado del país & 8.27 & Técnico & 8.27 \\
\hline \multirow{3}{*}{$\begin{array}{l}\text { Sexo } \\
\text { Femenino }\end{array}$} & $\%$ & Años residiendo en Cozumel & $\%$ & Relación con turismo & $\%$ \\
\hline & & & & & \\
\hline & 45.49 & Menos de 5 años & 8.65 & Trabaja en turismo & 37.59 \\
\hline & 52.63 & de 5 a 14 años & 15.04 & $\begin{array}{l}\text { Trabaja en actividad } \\
\text { relacionada al turismo }\end{array}$ & 28.20 \\
\hline \multirow{4}{*}{ Masculino } & & de 15 a 24 años & 25.94 & $\begin{array}{l}\text { Trabaja en actividad no } \\
\text { relacionada al turismo }\end{array}$ & 24.44 \\
\hline & & de 25 a 39 años & 29.70 & No trabaja & 9.40 \\
\hline & & de 40 a 59 años & 19.92 & & \\
\hline & & 60 a más años & 0.38 & & \\
\hline
\end{tabular}

Fuente: Elaboración propia

En la tabla 5 se observan los valores obtenidos en la encuesta para las 10 variables latentes evaluadas, en escala de 1 a 5 puntos, siendo éste último el mayor valor para cada variable. Se puede observar que se encontraron valores por arriba de la media teórica (3.0) para todas las variables latentes, con excepción notable de una de ellas: la dimensión del capital social estructural, que presentó media de 2.20. Por lo tanto se puede afirmar que, en general los valores fueron altos con excepción del capital social estructural que tuvo valor bajo. En general los encuestados perciben que en Cozumel se goza de una alta calidad de vida y que cuentan con capital social alto en sus dimensiones cognitiva y aptitudinal, no así en su dimensión estructural en la que se perciben con capital social bajo.

En los valores obtenidos para las variables latentes, se pueden destacar, para la calidad de vida, el valor más alto fue el de la satisfacción en el dominio de seguridad y salud con media de 4.42 y el valor más bajo se encontró en la satisfacción en el dominio de bienestar material, con media 3.5. Para el capital social, el más bajo fue la dimensión estructural ya mencionada y el valor más alto se encontró en la variable ajuste social del dominio aptitudinal con media 3.86. 
Tabla 5. Valores descriptivos de las variables de interés

\begin{tabular}{|c|c|c|c|c|c|}
\hline Variable & Media & $\begin{array}{l}\text { Desviación } \\
\text { estándar }\end{array}$ & Variable & Media & $\begin{array}{l}\text { Desviación } \\
\text { estándar }\end{array}$ \\
\hline Seguridad y Salud & 4.42 & 0.87 & Capital Social Cognitivo & 3.58 & 1.08 \\
\hline Bienestar Material & 3.50 & 1.13 & Capital Social Estructural & 2.20 & 1.31 \\
\hline Bienestar Emocional & 3.52 & 1.22 & Percepción Social & 3.51 & 1.12 \\
\hline $\begin{array}{l}\text { Bienestar } \\
\text { Medioambiental }\end{array}$ & 4.13 & 0.96 & Ajuste Social & 3.86 & 1.12 \\
\hline Bienestar Comunitario & 3.95 & 1.00 & & & \\
\hline Satisfacción global & 3.94 & 1.03 & & & \\
\hline
\end{tabular}

Fuente: Elaboración propia

\section{Contraste de hipótesis y discusión de los resultados}

Una vez realizada la validación de la escala, se ajustó un modelo de ecuaciones estructurales, mediante estructuras de covarianza (CB-SEM), del que se muestra una síntesis de los resultados en la tabla 6 . El ajuste del modelo se considera aceptable con valores de CFI y TLI cercanos al umbral de 0.9, con RMSEA de 0.073, dentro del intervalo de 0.05-0.1.

De acuerdo con los hallazgos en el estudio, se observa que la influencia del capital social estructural en la calidad de vida, solo se presenta en el caso del bienestar medioambiental, en tanto que las influencias hipotetizadas en el resto de los dominios resultaron rechazadas. Esto se puede interpretar en el sentido de que, en este sitio turístico, los residentes manifiestan contar con bajo nivel de capital estructural, es decir con poca participación en actividades comunitarias y por ende un bajo involucramiento con la sociedad local, sin embargo, esto no impacta en todos los dominios de su calidad de vida. Es posible que en el caso de Cozumel, la relación capital social estructural - calidad de vida sea moderada por alguna otra variable, tal pudiera ser el caso del lugar de nacimiento, dado el alto porcentaje de inmigrados, que probablemente no estén generando relaciones de colaboración con los nativos o con otros grupos de inmigrados.

Se encontró evidencia empírica para confirmar la influencia directa y positiva del capital social cognitivo, sobre cuatro de los cinco dominios de la satisfacción con la vida $\left(\mathrm{H}_{2}\right)$. Se confirmó la influencia del capital social cognitivo en cuatro de los cinco dominios de la calidad de vida, el bienestar comunitario, el bienestar emocional, el bienestar material y el bienestar en seguridad y salud. No se encontró evidencia de que el capital social cognitivo tuviera impacto en uno de los dominios de la calidad de vida, el bienestar medioambiental.

Esto se puede interpretar en el sentido de que, en el caso de Cozumel, la población residente considera que la confianza y armonía hacia sus pares en la comunidad, favorecen la satisfacción con la vida en los dominios comunitario, emocional, de salud y seguridad y material, sin embargo, no lo hace así en relación con su entorno natural inmediato. Es posible que la relación entre el capital social cognitivo y el bienestar medioambiental sea moderada por 
Tabla 6. Contraste de hipótesis

\begin{tabular}{|c|c|c|c|c|}
\hline Hipótesis & $\begin{array}{c}6 \\
\text { estandarizada }\end{array}$ & $\boldsymbol{t}$ & $P(>|z|)$ & Decisión \\
\hline $\begin{array}{l}\text { H1a: Capital social estructural } \rightarrow \text { Bienestar } \\
\text { comunitario }\end{array}$ & -0.041 & -0.567 & 0.571 & Rechazada \\
\hline $\begin{array}{l}\text { H1b: Capital social estructural } \rightarrow \text { Bienestar } \\
\text { emocional }\end{array}$ & -0.085 & -1.137 & 0.126 & Rechazada \\
\hline H1c: Capital social estructural $\rightarrow$ Bienestar material & -0.024 & -0.348 & 0.728 & Rechazada \\
\hline $\begin{array}{l}\text { H1d: Capital social estructural } \rightarrow \text { Bienestar } \\
\text { medioambiental }\end{array}$ & -0.117 & -1.722 & 0.085 & Aceptada \\
\hline H1e: Capital social estructural $\rightarrow$ Seguridad y salud & -0.085 & -1.137 & 0.256 & Rechazada \\
\hline $\begin{array}{l}\text { H2a: Capital social cognitivo } \rightarrow \text { Bienestar } \\
\text { comunitario }\end{array}$ & 0.299 & 3.688 & 0.000 & Aceptada \\
\hline H2b: Capital social cognitivo $\rightarrow$ Bienestar emocional & 0.315 & 4.279 & 0.000 & Aceptada \\
\hline H2c: Capital social cognitivo $\rightarrow$ Bienestar material & 0.192 & 2.544 & 0.011 & Aceptada \\
\hline $\begin{array}{l}\mathrm{H} 2 \mathrm{~d} \text { : Capital social cognitivo } \rightarrow \text { Bienestar } \\
\text { medioambiental }\end{array}$ & 0.084 & 1.17 & 0.242 & Rechazada \\
\hline H2e: Capital social cognitivo $\rightarrow$ Seguridad y salud & 0.233 & 2.794 & 0.005 & Aceptada \\
\hline H3a: Percepción social $\rightarrow$ Bienestar comunitario & -0.020 & -0.218 & 0.828 & Rechazada \\
\hline H3b: Percepción social $\rightarrow$ Bienestar emocional & 0.234 & 2.674 & 0.007 & Aceptada \\
\hline H3c: Percepción social $\rightarrow$ Bienestar material & 0.227 & 2.450 & 0.014 & Aceptada \\
\hline H3d: Percepción social $\rightarrow$ Bienestar medioambiental & -0.083 & -0.936 & 0.349 & Rechazada \\
\hline H3e: Percepción social $\rightarrow$ Seguridad y salud & -0.073 & -0.756 & 0.450 & Rechazada \\
\hline H4a: Ajuste social $\rightarrow$ Bienestar comunitario & 0.145 & 1.526 & 0.127 & Rechazada \\
\hline H4b: Ajuste social $\rightarrow$ Bienestar emocional & 0.112 & 1.297 & 0.194 & Rechazada \\
\hline H4c: Ajuste social $\rightarrow$ Bienestar material & 0.147 & 1.618 & 0.106 & Rechazada \\
\hline H4d: Ajuste social $\rightarrow$ Bienestar medioambiental & 0.436 & 4.620 & 0.000 & Aceptada \\
\hline H4e: Ajuste social $\rightarrow$ Seguridad y salud & 0.389 & 3.702 & 0.000 & Aceptada \\
\hline H5: Bienestar comunitario $\rightarrow$ Satisfacción con la vida & -0.033 & -0.467 & 0.641 & Rechazada \\
\hline H6: Bienestar emocional $\rightarrow$ Satisfacción con la vida & 0.127 & 1.818 & 0.069 & Aceptada \\
\hline H7: Bienestar material $\rightarrow$ Satisfacción con la vida & 0.393 & 4.670 & 0.000 & Aceptada \\
\hline $\begin{array}{l}\text { H8: Bienestar medioambiental } \rightarrow \text { Satisfacción con } \\
\text { la vida }\end{array}$ & 0.206 & 2.888 & 0.004 & Aceptada \\
\hline H9: Seguridad y salud $\rightarrow$ Satisfacción con la vida & 0.534 & 5.117 & 0.000 & Aceptada \\
\hline
\end{tabular}

$\chi^{2}(293)=702.362 * ; \mathrm{CFI}=0.906 ; \mathrm{TLI}=0.888 ; \operatorname{RMSEA}(90 \% \mathrm{IC})=0.073(0.066 ; 0.08)$

$* \mathrm{p}<0.01$

Fuente: Elaboración propia

alguna otra variable, que muy bien pudiera estar vinculada con los impactos de la actividad turística.

Respecto a las habilidades sociales, consideradas como dimensiones del capital social, los resultados fueron similares, en ambos casos se encontró evidencia empírica de influencia directa y positiva, sobre dos de los cinco dominios de la calidad de vida. En el caso de la 
percepción social, en los dominios de bienestar emocional y material, y en el caso del ajuste social en los dominios de bienestar medioambiental y del bienestar con seguridad y salud. Estos resultados pueden interpretarse en el sentido de que, si bien las habilidades sociales para la población residente en Cozumel constituyen un factor importante en la percepción de su calidad de vida, no lo son en todos sus dominios.

De los cinco dominios de la satisfacción con la vida, en la población residente de Cozumel, cuatro influyen de manera directa y positiva en la satisfacción global con la vida. La población de Cozumel percibe una mejoría en su calidad de vida, en la medida en que incremente el bienestar en sus dominios medioambiental, material, emocional y de seguridad y salud, lo cual es consistente con lo que teóricamente se esperaría. No obstante, para el caso del dominio del bienestar comunitario, no se encontró evidencia en favor de tal influencia, lo cual se puede interpretar en el sentido de que, las condiciones de índole comunitario tales como el transporte, los servicios públicos o los vecinos, no resultan tan relevantes para la satisfacción global con la vida para los habitantes de Cozumel, o que no lo son en la misma medida, o en el mismo sentido para todos ellos. Del mismo modo que en las reflexiones antes expuestas, para otras relaciones hipotéticas para las que no se halló evidencia estadísticamente significativa, es posible que existan variables moderadoras de la relación entre ambas variables latentes.

\section{CONCLUSIONES Y LÍNEAS FUTURAS DE INVESTIGACIÓN}

El turismo como toda actividad económica que se introduce en un territorio y que crece de manera acelerada, impacta en la vida de quienes residen en él (Monterrubio, 2018; King, et al., 1993), su crecimiento entre otros impactos, en lo social, económico y ambiental, genera crecimiento demográfico por inmigración, a grado tal que, como se encontró en el caso estudiado establece un contexto en el que la mitad de la población declara haber nacido en otro sitio. Esta condición, sin duda impacta en el tejido social, en las relaciones entre vecinos, en las redes de colaboración y confianza, así como también en la cultura, las instituciones y muy probablemente hasta en la ideología, los valores, y la idiosincrasia de los residentes. Este hecho no se encuentra documentado con detalle en la literatura turística, si bien existe profusa investigación sobre los impactos del turismo (Deery, et al., 2012, Sharma, et al., 2008) y entre ellos se ha documentado su influencia tanto en el capital social de los residentes (Moscardo, et al., 2017; Park, et al., 2012; Rodríguez y Vanesste, 2018), como en la calidad de vida (Andereck y Nyaupane, 2011; Kim, et al., 2013; Uysal, et al., 2012; Uysal, et al., 2016), ambos como aspectos considerados en el rubro de los impactos sociales del turismo, no se incluye en los análisis cómo interactúan éstas con el fenómeno migratorio. El presente estudio se centró en el análisis de la relación entre el capital social y la calidad de vida y si bien en su diseño no se incluyeron variables vinculadas a la inmigración, los resultados si resultan sugestivos, respecto de la necesidad de considerarla, como aspecto central al analizar la relación capital social calidad de vida.

Con base en esta reflexión, se puede afirmar que la principal contribución del presente estudio al conocimiento turístico consiste en aportar información empírica, que confirma la influencia del capital social en la calidad de vida en sitios turísticos, y al mismo tiempo, abre 
la posibilidad de que tal influencia no resulte necesariamente directa, sino mediada por otras variables.

En suma, las relaciones interpersonales, las redes de colaboración, solidaridad y cooperación surgidas de ellas y basadas en la confianza, el respeto entre vecinos y miembros de una comunidad aportan capital a los miembros de la comunidad en un sitio turístico; capital que considerado en tres dimensiones constitutivas, en términos de motivación, oportunidad y habilidad, influye directamente en la percepción de una mejor calidad de vida, lo cual se constató, al menos parcialmente, en el caso de la población residente en Cozumel.

Para efectos de la gestión del turismo en Cozumel, con los resultados obtenidos en este trabajo, se destaca la necesidad de acentuar la atención en acciones, que contribuyan a mejorar las condiciones del capital social a nivel comunitario, especialmente aquellas orientadas al fortalecimiento de las redes de colaboración y las habilidades para aprovechar tales redes entre la población, pues en la medida en la que estas se fortalezcan, también mejorará la percepción subjetiva de la calidad de vida en la isla.

Una aportación adicional del presente trabajo se encuentra en los instrumentos utilizados para medir el capital social, que arrojaron resultados válidos tanto en constructo como en sus valores de fiabilidad compuesta y validez convergente, lo que permite no sólo hacer uso de ellos para reportar los valores que se encontraron para el caso estudiado, sino permiten proponerlos a su vez para ser utilizados en contextos o condiciones similares. Por otra parte, salta a la vista el hecho de que el capital social estructural obtuvo una valoración baja para el caso de la población residente en Cozumel, lo que refleja una relativamente baja participación en colectivo, lo que a su vez impacta en una menor satisfacción con la calidad de vida.

En relación con las limitaciones del estudio, cabe destacar por una parte que, la influencia de la satisfacción con cinco dimensiones del bienestar, en la satisfacción global con la vida, se considera en la literatura especializada como una adecuada aproximación a medida de la calidad de vida percibida subjetivamente. Sin embargo en el estudio realizado, para el caso de la población de Cozumel, no se encontró evidencia de que el dominio del bienestar comunitario influyera de manera significativa en la satisfacción global, este hallazgo conduce a la reflexión respecto a la necesidad de analizar en detalle esta relación, ya sea mediante nuevas aproximaciones cuantitativas con instrumentos más afinados y detallados, o bien a través de una aproximación con herramientas cualitativas, en búsqueda de variables o factores que moderen la relación. Asimismo, no se puede descartar la necesidad de revisar los instrumentos utilizados para mensurar la calidad de vida, ya que por una parte, la eliminación de algunos ítems que se efectuó con el fin de mejorar el ajuste del modelo, así como los propios valores finales de ajuste que, si bien fueron aceptables, son evidentemente susceptibles de mejora para futuras investigaciones.

El hecho de que se encontrara que varias de las relaciones hipotetizadas no fueran confirmadas empíricamente en el caso analizado apunta hacia la necesidad de realizar nuevos estudios en el mismo y en otros casos, respecto a la relación entre el capital social, particularmente en sus dimensiones estructural y de habilidades sociales (Adler y Kwon, 2002; Kwon y Adler, 2014), sobre la calidad de vida de la población, así como la exploración de los efectos moderadores de otras variables como, sin ser exhaustivos, la inmigración al sitio turístico, el 
grado de relación con la actividad turística, la edad y el sexo, los niveles de ingresos. Además, los hallazgos permiten plantear la necesidad de analizar las relaciones del capital social, con otros factores de la percepción subjetiva de la calidad de vida, tales como los rasgos socioculturales y de personalidad, el rol que juegan las emociones sociales, las ideologías y creencias de los grupos e individuos que residen en los sitios turísticos, así como sus relaciones con otras variables, entre las que destaca la inmigración, atraída por el auge económico del desarrollo turístico y por las propias condiciones atractivas del sitio turístico.

Por último, pero no menos importante, señalar la necesidad de continuar analizando la calidad de vida, vinculada a otras variables o indicadores del buen vivir, el bienestar, la psicología positiva, la felicidad, la plenitud en la vida (Hidalgo y Cubillo, 2014) y sus potenciales aportaciones al desarrollo sostenible de las comunidades receptoras de turismo.

Para finalizar, se pueden delinear algunas recomendaciones prácticas para el sector turismo en Cozumel, de manera que sus impactos incidan favorablemente en la percepción subjetiva de la calidad de vida entre la población:

Dado que los impactos del turismo en el sitio de destino, además de los económicos, son relevantes los sociales para la calidad de vida de la población local, resulta recomendable que como parte de los planes de desarrollo a nivel local, ya sean los impulsados por la administración municipal o por otras instituciones u organizaciones privadas o mixtas, es necesario incluir estrategias y acciones para incentivar el desarrollo del capital social de la población residente en el destino.

Del mismo modo, como parte de las acciones de gestión y evaluación del turismo en el ámbito local en los destinos turísticos como el caso de Cozumel, se hace relevante la generación de indicadores, así como su monitoreo, sobre el capital social y la calidad de vida de la población. Este monitoreo además de generar una base de datos útil para la planificación, permitiría también tomar acciones preventivas y correctivas a autoridades locales.

Por su parte, el sector empresarial del turismo en Cozumel, que se beneficia directamente del turismo y de su desarrollo en el largo plazo, puede impulsar acciones para incentivar el desarrollo del capital social entre su fuerza laboral y sus familias, de modo que incida también en la percepción de mejora en su calidad de vida.

De manera intensiva, las organizaciones de la sociedad civil, pueden reforzar su actividad a nivel de desarrollo comunitario, incentivando acciones para el desarrollo del capital social entre la población y con ello a su vez, también incidir favorablemente en la calidad de vida. 


\section{REFERENCIAS BIBLIOGRÁFICAS}

Adler, P. S., y Kwon, S.-W. (2002). Social capital: prospects for a new concept. Academy of Management Review, 27 (1), 17-40. http://doi.org/10.5465/AMR.2002.5922314

Aldás Manzano, J., y Uriel Jiménez, E. (2017). Análisis multivariante aplicado con R. Paraninfo.

Allen, L., y Gibson, R. (2009). Perceptions of Community Life and Services: a Comparison between Leaders and Community Residents. Community Development Society Journal, 18 (1), 89-103. https://doi.org/10.1080/15575338709490037

Aminzadeh, K., Denny, S., Utter, J., Milfont, T.L., Ameratunga, S., Teevale, T., Clark, T., 2013. Neighbourhood social capital and adolescent self-reported wellbeing in New Zealand: a multilevel analysis. Social Science Medical. 84, 13-21.

Andereck, K. L., y Nyaupane, G. P. (2011). Exploring the Nature of Tourism and Quality of Life Perceptions among Residents. Journal of Travel Research, 50 (3), 248-260. https://doi. org/10.1177/0047287510362918

Ap, J., y Crompton, J. L. (1993). Residents' Strategies for Responding to Tourism Impacts. Journal of Travel Research, 32 (1), 47-50. http://doi.org/https://doi.org/10.1177 \%2F004728759303200108

Ap, J., y Crompton, J. L. (1998). Developing and Testing a Tourism Impact Scale. Journal of Travel Research, 37 (November 1998), 120-130.

Baker, W., y Faulkner, R. R. (2009). Social Capital, Double Embeddedness, and Mechanisms of Stability and Change. American Behavioral Scientist, 52 (11), 1531-1555. http://doi. org/10.1177/0002764209331525

Baron, R. A., y Markman, G. D. (2003). Beyond social capital: the role of entrepreneurs' social competence in their financial success. Journal of Business Venturing, 18 (1), 41-60. http://doi.org/10.1016/S0883-9026(00)00069-0

Berkman, L., y Syme, S. L. (1979). Social networks, host resistance and mortality. American Journal of Epidemiology, 109, 186-204.

Blumberg, M., y Pringle, C. D. (1986). The Missing Opportunity in Organizational Research: Some Implications for a Theory of Work Performance. Academy of Management Review, 7 (4), 560-569. http://doi.org/10.5465/AMR.1982.4285240

Bojórquez, J., López, L., Hernández, M., y López, E. (2013). Utilización del alfa de Cronbach para validar la confiabilidad de un instrumento de medición de satisfacción del estudiante en el uso del software Minitab. Laccei, 1-24. https://doi.org/10.1016/ S0015-7368(91)73199-X

Bonnes, M., Bonaiuto, M., y Ercolani, A. P. (1991). Crowding and Residential Satisfaction in the Urban Environment. Environment and Behavior, 23 (5), 531-552. https://doi. org/10.1177/0013916591235001

Bourdieu, P. (1980). Le capital social. Actes de La Recherche En Sciences Sociales, 31 (31), 1-3. http://doi.org/10.7328/jurpcb201328577

Briggs, D. (2010) 'True stories from bare times on road': developing empowerment, identity and social capital among urban minority ethnic young people in London, UK. Ethnographic Racial Studies. 33 (5), 851-871. http://dx.doi.org/10.1080/01419870903254687

Bruin, M. J., y Cook, C. C. (1997). Understanding Constraints and Residential Satisfaction Among Low-Income Single-Parent Families. Environment and Behavior, 29 (4), 532553. https://doi.org/10.1177/001391659702900405 
Butler, R. W. (1980). The concept of a tourist area cycle of evolution: implications for management of resources. The Canadian Geographer/Le Géographe Canadien, 24(1), 5-12.

Camfield, L., y Skevington, S. M. (2008). On subjective well-being and quality of life. Journal of Health Psychology, 13 (6), 764-775. https://doi.org/10.1177/1359105308093860

Carpiano, R.M. (2007) Neighborhood social capital and adult health: an empirical test of a Bourdieu-based model. Health Place 13, 639-655

Casanueva, C., Gallego, Á. y Sancho, M., (2013) Network resources and social capital in airline alliance portfolios. Tourism Management, 36, 441-453.

Castillo, D., Aguirre, C., y Romero, F. (2015). Turismo Un Aporte Al Buen Vivir Comunitario En Territorios Andinos Rurales Ecuatorianos. Nariz Del Diablo. European Scientific Journal, $11(20), 88-105$.

Chesney, M., y Hazari, B. R. (2003). Illegal Migrants, Tourism and Welfare: A Trade Theoretic Approach. Pacific Economic Review, 8 (3), 259-268.

Choi, H.C., y Murray, I. (2010). Resident attitudes toward sustainable community tourism. Journal of Sustainable Tourism, 18 (4), 575-594. http://doi.org/10.1080/09669580903524852

Coleman, J. S. (1988). Social capital in the creation of human capital. The American Journal of Sociology, 94 (Supplement: Organizations and Institutions), S95-S120. https://doi. org/10.1037/0012-1649.22.6.723

Coleman, J. S. (1990) Foundations of social theory. Cambridge: Harvard University Press.

Coleman, J. S., y Coleman, J. S. (1994). Foundations of social theory. Harvard university press.

Cortina, J.M. (1993). What is coefficient al pha? An examination of theoryand applications. Journal of Applied Psychology, 78 (1), 98-104. https://doi.org/10.1037/0021-9010.78.1.98

Dai, W. D., Mao, Z. E., Zhao, X. R., y Mattila, A. S. (2015). How does social capital influence the hospitality firm's financial performance? The moderating role of entrepreneurial activities. International Journal of Hospitality Management, 51, 42-55. https://doi. org/10.1016/j.ijhm.2015.08.011

Deery, M., Jago, L., y Fredline, L. (2012). Rethinking social impacts of tourism research: A new research agenda. Tourism Management, 33 (1), 64-73. http://doi.org/10.1016/j. tourman.2011.01.026

Diener, E., Lucas, R., Schimmack, U. y Helliwell, J. (2009) Well-being for Public Policy. Oxford University Press, Oxford.

Diener, E., Suh, E. M., Lucas, R. E., y Smith, H. L. (1999). Subjective Well-Being: Three Decades of Progress. Psychological Bulletin, 125 (2), 276-302.

Doxey, G. (1975). A causation theory of visitor-resident irritants: Methodology and research inferences. En the impact of tourism sixth annual conference proc of the travel research Association.

Doğan, H. Z. (1989). Forms of adjustment. Sociocultural impacts of tourism. Annals of Tourism Research, 16 (2), 216-236. http://doi.org/10.1016/0160-7383(89)90069-8

Durkheim, E. (1971). El suicidio: estudio de sociología. Editorial Akai. Madrid.

Elgar, F. J., Davis, C. G., Wohl, M. J., Trites, S. J., Zelenski, J. M., y Martin, M. S. (2011). Social capital, health and life satisfaction in 50 countries. Health and Place, 17 (5), 1044-1053. https://doi.org/10.1016/i.healthplace.2011.06.010

Eriksson, M., 2011. Social capital and health-implications for health promotion. Global Health Action, 4. 
Faulkner, B., Tideswell, C., Faulkner, B., y Tideswell, C. (1997). A Framework for Monitoring Community Impacts of Tourism A Framework for Monitoring Community Impacts of Tourism. Journal of Sustainable Tourism, 5 (1), 3-28. http://doi. org/10.1080/09669589708667273

Fayers, P. M., y Hand, D. J. (1997). Factor analysis, causal indicators and quality of life. Quality of Life Research: An International Journal of Quality of Life Aspects of Treatment, Care and Rehabilitation, 6(2), 139-150. https://doi.org/10.1023/A:1026490117121

Frauman, E., y Banks, S. (2011). Gateway community resident perceptions of tourism development: Incorporating Importance-Performance Analysis into a Limits of Acceptable Change framework. Tourism Management, 32 (1), 128-140. http://doi.org/10.1016/i. tourman.2010.01.013

Fredline, L. (2002). Social impacts of tourism on the Gold Coast. Gold Coast Tourism Visioning Project 3.3. Australia.

Gabbay, S. M., y Leenders, R. Th. A. J. (1999). CSC: The structure of advantage and disadvantage. En R. Th. A. J. Leenders y S. M. Gabbay (Eds.), Corporate social capital and liability: 1-14. Boston: Kluwer.

Galster, G. C., y Hesser, G. W. (1981). Residential Satisfaction. Environment and Behavior, 13 (6), 735-758. https://doi.org/10.1177/0013916581136006

García, E. D., y González, A. (2018). Relaciones sociales duraderas entre tripulación de cruceros y población residente, análisis del caso Cozumel desde la teoría del intercambio social. PASOS Revista de Turismo y Patrimonio Cultural, 16 (4), 1089-1104.

García-Villaverde, P. M., Elche, D., Martínez-Pérez, Á., y Ruiz-Hortega, M. J. (2017). Determinants of radical innovation in clustered firms of the hospitality and tourism industry. International Journal of Hospitality Management, 61, 45-58. https://doi.org/10.1016/i. ijhm.2016.11.002

González, A., Pacheco, M. Y., y Macías, A. R. (2016). Influencia de la participación comunitaria y la percepción de beneficios económicos del turismo en el capital social y comportamiento pro-ambiental. Análisis estructural en comunidades mayas rurales de Quintana Roo, México. En A. E. Gámez, A. Palafox Muñoz, y M. Gutiérrez (Eds.), La Construcción del Espacio Turístico. Procesos, Actores e Impactos. Memorias del IX Congreso de la AMIT (pp. 457-475). La Paz: AMIT.

González, A., y Herrera, H. R. (2017). Informe de Diagnóstico Comunitario Participativo Cozumel 2016. Cozumel.

González, A., y Macías, A. R. (2017). Impactos de los viajes de visita a familiares y amigos y su influencia en la calidad de vida de la población residente en Cozumel, México. En XI Congreso Internacional de la AMIT (pp. 1-19). Guadalajara: Academia Mexicana de Investigación Turística A.C.

González, M., y Iglesias, G. (2009). Impactos del turismo sobre los procesos de cohesión social: Caibarién, Cuba. Estudios y Perspectivas En Turismo, 18 (1), 53-68.

Granovetter, M. (1992). Economic Institutions as Social Constructions: A Framework for Analysis. Acta Sociologica, 35 (1), 3-11. http://doi.org/10.1177/000169939203500101

Hamdan, H., Yusof, F., y Marzukhi, M. A. (2014). Social Capital and Quality of Life in Urban Neighborhoods High Density Housing. Procedia - Social and Behavioral Sciences, 153, 169-179. https://doi.org/10.1016/i.sbspro.2014.10.051 
Hausman, A.J., Becker, J. y Brawer, R. (2005) Identifying value indicators and social capital in community health partnerships. J. Community Psychology. 33 (6), 691-703

Hidalgo, A., y Cubillo, A. (2014). Seis debates abiertos sobre el sumak kawsay. Íconos: Revista de Ciencias Sociales, 48 (1), 25-40.

Häuberer, J. (2011). Social Capital Theory. Towards a Methodological Foundation (1a ed.). Wiesbaden: VS Research.

Inaba, Y., Wada, Y., Ichida, Y., y Nishikawa, M. (2015). Which part of community social capital is related to life satisfaction and self-rated health? A multilevel analysis based on a nationwide mail survey in Japan. Social Science and Medicine, 142, 169-182. https:// doi.org/10.1016/i.socscimed.2015.08.007

INEGI (2018) Consulta interactiva de datos. Consultada el 12 de Noviembre, 2018, de www3. inegi.org.mx

INEGI. (2010). Censo Nacional de Población y Vivienda. INEGI: México.

INEGI. (2015). Mapas de INEGI. Consultada el 25 de Septiembre, 2018, de http://www.beta. inegi.org.mx/app/mapas/

Kawachi, I., Berkman, L.F. (2000) Social cohesion, social capital, and health. En: Berkman, L.F., Kawachi, I. (Eds.), Social Epidemiology. Oxford University Press, pp.

Kay Smith, M., y Diekmann, A. (2017). Tourism and wellbeing. Annals of Tourism Research, 66, 1-13. http://doi.org/10.1016/i.annals.2017.05.006

Kim, D., y Kawachi, I. (2007). U.S. State-Level Social Capital and Health-Related Quality of Life: Multilevel Evidence of Main, Mediating, and Modifying Effects. Annals of Epidemiology, 17(4), 258-269. http://doi.org/10.1016/j.annepidem.2006.10.002

Kim, K., Uysal, M., y Sirgy, M. J. (2013). How does tourism in a community impact the quality of life of community residents? Tourism Management, 36, 527-540. http://doi. org/10.1016/j.tourman.2012.09.005

King, B., Pizam, A., y Milman, A. (1993). Social impacts of tourism. The Routledge Handbook of Tourism Impacts, 20, 650-665. https://doi.org/10.4324/9781351025102-18

Kwon, S.-W., y Adler, P. S. (2014). Social Capital: Maturation of a Field of Research. Academy of Management Review, 39 (4), 412-422. http://doi.org/10.5465/amr.2014.0210

Land, K., Michalos, A., y Sirgy, M. (2011). Handbook of social indicators and quality of life research. Springer y Business Media.

Lee, S.-Y., Song, X.-Y., Skevington, S. M., y Hao, Y.-T. (2005). Application of structural equation models to quality of life. Structural Equation Modeling, 12 (3), 411-434. https://doi. org/10.1207/s15328007sem 1203

Lee, Y. J. (2008). Subjective quality of life measurement in Taipei. Building and Environment, 43 (7), 1205-1215. https://doi.org/10.1016/i.buildenv.2006.11.023

Li, Y., y Hunter, C. (2015). Community involvement for sustainable heritage tourism: a conceptual model. Journal of Cultural Heritage Management and Sustainable Development, 5 (3), 248-262. http://doi.org/10.1108/JCHMSD-08-2014-0027

Lin, N. (1999) Social networks and status attainment. Annual Review of Sociology, 25: 467-87

Lin, N. (2001). Social capital: a theory of social structure and action. Cambridge: Cambridge University Press. 
Liu, J., Qu, H., Huang, D., Chen, G., Yue, X., Zhao, X., y Liang, Z. (2014). The role of social capital in encouraging residents' pro-environmental behaviors in community-based ecotourism. Tourism Management, 41, 190-201. http://doi.org/10.1016/j.tourman.2013.08.016

Lochner, K. A., Kawachi, I., Brennan, R. T., y Buka, S. L. (2003). Social capital and neighborhood mortality rates in Chicago. Social Science \& Medicine, 56 (8). 1797-1805.

Lu, M. (1999). Determinants of Residential Satisfaction: Ordered Logit vs. Regression Models. Growth and Change, 30 (2), 264-287. https://doi.org/10.1111/0017-4815.00113

Lyubomirsky, S., King, L., y Diener, E. (2005). The benefits of frequent positive affect: Does happiness lead to success? Psychological Bulletin, 131 (6), 803-855. https://doi. org/10.1037/0033-2909.131.6.803

Maass, R., Kloeckner, C. A., Lindstrøm, B., y Lillefjell, M. (2016). The impact of neighborhood social capital on life satisfaction and self-rated health: A possible pathway for health promotion? Health and Place, 42(October), 120-128. https://doi.org/10.1016/i. healthplace.2016.09.011

Martínez-Pérez, Á., García-Villaverde, P. M., y Elche, D. (2016). The mediating effect of ambidextrous knowledge strategy between social capital and innovation of cultural tourism clusters firms. International Journal of Contemporary Hospitality Management, 28 (7), 1484-1507. https://doi.org/10.1108/IJCHM-08-2014-0405

McCabe, S., y Johnson, S. (2013). The happiness factor in tourism: Subjective well-being and social tourism. Annals of Tourism Research, 41, 42-65. http://doi.org/10.1016/i. annals.2012.12.001

McGehee, N. G., Lee, S., O'Bannon, T. L., y Perdue, R. R. (2010). Tourism-related Social Capital and Its Relationship with Other Forms of Capital: An Exploratory Study. Journal of Travel Research, 49(4), 486-500. http://doi.org/10.1177/0047287509349271

Merinero, R. (2008). Micro-Cluster Turísticos El Papel Del Capital Social En El Desarrollo Económico Local. Revista de Estudios Empresariales, 2, 67-92.

Mohnen, S.M., Volker, B., Flap, H. y Groenewegen, P. P. (2012) Health-related behavior as a mechanism behind the relationship between neighborhood social capital and individual health - a multilevel analysis. BMC Public Health 12 (116). http:// dx.doi. org/10.1186/1471-2458-12-116

Monterrubio, J. C. (2008). Residents ' Perception of Tourism : A Critical Theoretical and Methodological Review. Ciencia Ergo Sum, 15 (1), 35-44.

Monterrubio, J. C. (2018). Impactos socioculturales del turismo (1a ed.). México: Trillas.

Moran, P. (2005). Structural vs. relational embeddedness: social capital and managerial performance. Strategic Management Journal, 26 (12), 1129-1151. https://doi.org/10.1002/ smj. 486

Moscardo, G. (2009). Tourism and Quality of Life: Towards a More Critical Approach. Tourism and Hospitality Research, 9(2), 159-170. http://doi.org/10.1057/thr.2009.6

Moscardo, G., Konovalov, E., Murphy, L., McGehee, N. G., y Schurmann, A. (2017). Linking tourism to social capital in destination communities. Journal of Destination Marketing and Management. https://doi.org/10.1016/i.jdmm.2017.10.001

Nahapiet, J., y Ghoshal, S. (1997). Social Capital, Intellectual Capital and the Creation of Value in Firms. Academy of Management Proceedings, 1997 (1), 35-39. https://doi. org/10.5465/ambpp.1997.4980592 
Nahapiet, J., y Ghoshal, S. (1998). Social capital, intellectual capital, and the organizational advantage. The Academy of Management Review, 23 (2), 242-266.

Nawijn, J., y Filep, S. (2016). Two directions for future tourist well-being research. Annals of Tourism Research, 61, 221-223. http://doi.org/10.1016/j.annals.2016.07.007

Nilsson, J., Rana, A. K. M. M., y Kabir, Z. N. (2006). Social Capital and Quality of Life in Old Age. JournalofAgingand Health, 18(3),419-434.http://doi.org/10.1177/0898264306286198

Nunkoo, R., y Gursoy, D. (2012). Residents' support for tourism. Annals of Tourism Research, 39(1), 243-268. http://doi.org/10.1016/i.annals.2011.05.006

Oliveira, M. C. B. de, y Pimentel, T. D. (2016). El Campo Turístico de la ciudad de Quito (Ecuador): un análisis del capital de sus agentes y de la (posible) acción colectiva sobre las políticas de turismo como objeto de disputa. Investigaciones Turísticas, (12), 183-209. http:// doi.org/10.14198/INTURI2016.12.09

Ostrom, E., y Ahn, T. K. (2009). The meaning of social capital and its link to collective action. En G. Tinggaard Svendsen y G. Lind Haase Svendsen (Eds.), Handbook of Social Capital. The Troika of Sociology, Political Science and Economics (1a ed., pp. 17-35). Cheltenham, UK: Edward Elgar Publishing Inc.

Park, D.-B., Lee, K.-W., Choi, H.-S., y Yoon, Y. (2012). Factors influencing social capital in rural tourism communities in South Korea. Tourism Management, 33 (6), 1511-1520. http:// doi.org/10.1016/i.tourman.2012.02.005

Pearson, A. W., Carr, J. C., y Shaw, J. C. (2008). Toward a Theory of Familiness: A social capital perspective. Entrepreneurship: Theory and Practice, 32 (6), 949-969.

Perez, A., y Camberos, M. (2017). Desigualdad, Turismo y Bienestar: Análisis regional y por estados, según estrato urbano y rural. El Periplo Sustentable, (32).

Pitchford, M. (2008). Making Spaces for Community Development. Bristol: Policy Press

Portes, A. (1998). Social Capital: Its Origins and Applications in Modern Sociology. Annual Review of Sociology, 24(1), 1-24. https://doi.org/10.1210/endo-59-4-454

Putnam, R. (1993). The prosperous community. The American Prospect. EUA.

Putnam, R. (1995). Bowling alone: America's declining social capital. Journal of Democracy.

R Core Team. (2018). R: A language and environment for statistical computing. Vienna, Austria: R Foundation for Statistical Computing. Recuperado de https://www.r-project.org/

Rablen, M.D., 2012. The promotion of local wellbeing: a primer for policymakers. Local Economics, 27, 297. http://dx.doi.org/10.1177/0269094211434488

Ramírez, O. I., Cruz, G., y Vargas, E. E. (2018). Un acercamiento al Capital Social y al Turismo desde el enfoque mixto y mapeo de actores. Revista de Antropología Experimental, 4, 55-73.

Rasoolimanesh, S. M., Ringle, C. M., Jaafar, M., y Ramayah, T. (2017). Urban vs. rural destinations: Residents' perceptions, community participation and support for tourism development. Tourism Management, 60, 147-158. http://doi.org/10.1016/i.tourman.2016.11.019

Requena, F. (2003). Social capital, satisfaction and quality of life in the workplace. Social Indicators Research, 61 (3), 331-360. http://doi.org/10.1023/A:1021923520951

Rodriguez, S., y Vanneste, D. (2018). Social capital at the tourist destination level: Determining the dimensions to assess and improve collective action in tourism. Tourist Studies. https://doi.org/10.1177/1468797618790109 
Rosseel, Y. (2012). lavaan: An R Package for Structural Equation Modeling. Journal Of Statistical Software, 48(2), 1-36.

Sandoval, L., y Ortega, M. E. (2018). Índice de capital social para el Pueblo Mágico de Tapijulapa, Tabasco Rate of Social Capital for the Magic Town of Tapijulapa, Tabasco. Revista de El Colegio de San Luis, VIII (15), 171-201.

Secretaría de Desarrollo Turístico del Estado de Quintana Roo. (2017). Perfil y comportamiento del turista en Cozumel Julio - Septiembre 2017. Recuperado de http://qroo.gob.mx/ sedetur/estudios-del-perfil-del-turista-en-quintana-roo-0

SECTUR, Gobierno del Estado de Quintana Roo, y Universidad de Quintana Roo. (2014). Agendas de Competitividad de los destinos turísticos de México. Cozumel, Quintana Roo. México.

Sharma, B., Dyer, P., Carter, J., y Gursoy, D. (2008). Exploring Residents' Perceptions of the Social Impacts of Tourism on the Sunshine Coast, Australia. International Journal of Hospitality y Tourism Administration, 9(3), 288-311. http://doi.org/10.1080/15256480802096092

Sharpley, R. (2014). Host perceptions of tourism: A review of the research. Tourism Management, 42 (1), 37-49. http://doi.org/10.1016/i.tourman.2013.10.007

Sirgy, M. (2012). The psychology of quality of life: Hedonic well-being, life satisfaction, and eudaimonia. Springer Science \& Business Media.

Sirgy, M. J., Rahtz, D. R., Cicic, M., Underwood, R., Social, S., y Mar, N. (2000). A method for assessing residents' satisfaction with community-based services: a quality-of-life perspective. Social Indicator Research, 49 (3), 279-316.

Sirgy, M. J., y Cornwell, T. (2001). Further validation of the Sirgy et al.'s measure of community quality of life. Social Indicators Research, 56 (2), 125-143.

Sirgy, M., y Cornwell, T. (2002). How neighborhood features affect quality of life. Social Indicators Research, 59 (1), 79-114.

Soares, J. C., y Gandara, J. M. (2012). Indicadores para analizar la evolución del ciclo de vida de los destinos turísticos litorales Investigaciones Turísticas Indicators to analyze the life cycle of coastal tourist destination. Investigaciones Turísticas, 3 (3), 19-38. http://doi. org/http://dx.doi.org/10.14198/INTURI2012.3.02

Subramanian, S. V., Kim, D. J., y Kawachi, I. (2002). Social trust and self- rated health in U.S. communities: A multilevel analysis. Journal of Urban Health-Bulletin of the New York Academy of Medicine, 79 (4), 21-34.

Taber, K. S. (2017). The Use of Cronbach's Alpha When Developing and Reporting Research Instruments in Science Education. Research in Science Education, 1-24. https://doi. org/10.1007/s11165-016-9602-2

Tang, L. R. (2014). The application of social psychology theories and concepts in hospitality and tourism studies: A review and research agenda. International Journal of Hospitality Management, 36, 188-196. https://doi.org/10.1016/i.ijhm.2013.09.003

Teye, V., Sirakaya, E., y F. Sönmez, S. (2002). Residents' attitudes toward tourism development. Annals of Tourism Research, 29 (3), 668-688. http://doi.org/10.1016/ $\underline{\mathrm{S} 0160-7383(01) 00074-3}$

Tsai, W., y Ghoshal, S. (1998). Social capital and value creationThe role of intrafirm networks. Academy of Management Journal, 41 (4), 464-476. https://doi.org/10.2307/257085 
Uphoff, N., y Langholz, J. (1998). Incentives for avoiding the tragedy of the commons. Environmental Conservation, 25 (3), 251-261.

Urtasun, A., y Gutiérrez, I. (2006). Tourism agglomeration and its impact on social welfare: An empirical approach to the Spanish case. Tourism Management, 27 (5), 901-912. http:// doi.org/10.1016/j.tourman.2005.05.004

Uysal, M., Perdue, R. R., Sirgy, M. J., y Joseph Sirgy, M. (2012). Handbook of tourism and quality-of-life research : enhancing the lives of tourists and residents of host communities. Handbook of Tourism and Quality-of-Life Research: Enhancing the Lives of Tourists and Residents of Host Communities. Springer. http://doi.org/10.1007/978-94-007-2288-0

Uysal, M., Sirgy, M., Woo, E., y Kim, H. (2016). Quality of life (QOL) and well-being research in tourism. Tourism Management, 53, 244-261. http://dx.doi.org/10.1016/i. tourman.2015.07.013

van Griethuijsen, R. A. L. F., van Eijck, M. W., Haste, H., den Brok, P. J., Skinner, N. C., Mansour, N., y BouJaoude, S. (2015). Global patterns in students' views of science and interest in science. Research in Science Education, 45 (4), 581-603. https://doi.org/10.1007/ s11165-014-9438-6

Veloso, E., y Delgado, M. (2008). A participação das comunidades autóctones no turismo e o capital social no processo de desenvolvimento local: um exame do Programa Turismo Solidário - MG. Caderno Virtual de Turismo, 8 (1), 1-15.

Vemuri, A.W., Grove, J.M., Wilson, M.A. y Bruch, W.R. (2011) A tale of two scales: evaluating the relationship among life satisfaction, social capital, income, and the natural environment at individual and neighborhood levels in metropolitan Baltimore. Environmental Behavior. 43 (1), 3-25. http://dx.doi.org/10.1177/0013916509338551.

Vrbka, J. S., y Combs, E. (1993). Predictors of Neighborhood and Community Satisfactions in Rural Communities. Housing and Society, 20 (1), 41-49. https://doi.org/10.1080/0888 $\underline{2746.1993 .11430153}$

Wen, M., Browning, C. y Cagney, K. (2003). Poverty affluence and income inequality: neighborhood economic structure and its implications for health. Social Science and Medicine $57,843-860$

Wiesmann, U. y Hannich, H.J. (2013) The contribution of resistance resources and Sense of Coherence to Life satisfaction in older age. Journal of Happiness Studies 14 (3). http:// dx.doi.org/10.1007/s10902-012-9361-3.

Woo, E., Kim, H., y Uysal, M. (2015). Life satisfaction and support for tourism development. Annals of Tourism Research, 50, 84-97. http://doi.org/10.1016/i.annals.2014.11.001

Woolcock, M. (2010). The Rise and Routinization of Social Capital, 1988-2008. Annual Review of Political Science, 13 (1), 469-487. http://doi.org/10.1146/annurev. polisci.031108.094151

Woosnam, K. M., Norman, W. C., y Ying, T. (2009). Exploring the theoretical framework of emotional solidarity between Residents and tourists. Journal of Travel Research, 48(2), 245-258. http://doi.org/10.1177/0047287509332334

Younsi, M., y Chakroun, M. (2016). Does social capital determine health? Empirical evidence from MENA countries. Social Science Journal, 53 (3), 371-379. https://doi.org/10.1016/i. soscij.2014.08.008 
Yu, C. P., Cole, S. T., y Chancellor, C. (2016). Assessing Community Quality of Life in the Context of Tourism Development. Applied Research in Quality of Life, 11(1), 147-162. http:// doi.org/10.1007/s11482-014-9359-6

Ziakas, V., y Costa, C. A. (2010). 'Between Theatre and Sport' in a Rural Event: Evolving Unity and Community Development from the Inside-Out. Journal of Sport y Tourism, 15 (1), 7-26. http://doi.org/10.1080/14775081003770892

Zoghbi-Manrique-de-Lara, P., Verano-Tacoronte, D., y Guerra-Báez, R. M. (2016). El bienestar y la conducta de los empleados de hotel en el contexto de la actual crisis económica. Investigaciones Turísticas, (11), 96-115. 